\title{
Complexity of Sizing for Space Suit Applications
}

\author{
Elizabeth Benson $^{1}$ and Sudhakar Rajulu ${ }^{2}$ \\ ${ }^{1}$ MEI Technologies 2525 Bay Area Blvd. \#300 Houston, TX 77058 \\ ${ }^{2}$ NASA Johnson Space Center, 2101 NASA Parkway Houston, TX \\ \{Elizabeth. Benson, Sudhakar.Rajulu-1\} @NASA.gov
}

\begin{abstract}
The 'fit' of a garment is often considered to be a subjective measure of garment quality. However, some experts attest that a complaint of poor garment fit is a symptom of inadequate or excessive ease, the space between the garment and the wearer. Fit has traditionally been hard to quantify, and space suits are an extreme example, where fit is difficult to measure but crucial for safety and operability. A proper space suit fit is particularly challenging because of NASA's desire to fit an incredibly diverse population (males and females from the $1^{\text {st }}$ to $99^{\text {th }}$ percentile) while developing a minimum number of space suit sizes. Because so few sizes are available, the available space suits must be optimized so that each fits a large segment of the population without compromising the fit of any one wearer.
\end{abstract}

\section{Introduction}

Successfully predicting wearer dimensions and providing the appropriate amount of slack and adjustability is crucial in developing space suits, where a poor fit can decrease mobility and lead to wearer discomfort or even injury. Suit designers need to know the sizes of the people they need to fit, the amount of adjustability the suits need, and how well a suit must fit to be usable. Additionally, it is important to make sure a suit fits before it is evaluated, or used to evaluate other systems. Therefore, the Anthropometry and Biomechanics Facility at NASA's Johnson Space Center is working in conjunction with the Pressure Garment Group at NASA to combine traditional and more advanced methods of quantifying fit, to aid the designers of the next generation of space suits. This paper describes the issues that are faced in attempting to fit suits to a diverse population, and some of the methods that can be used to surmount these difficulties and provide the best possible compromise between fit and accommodation.

\section{A Background on Suit Fit}

Past NASA suit systems have used a variety of techniques with varying success to fit their target populations. These have ranged from the custom sizing used in the Apollo program, to the off-the-shelf approach of the current Advanced Crew Escape Suit, which is an adjustable suit available in a set of standard sizes. 


\subsection{Custom Tailoring: The Apollo A7LB}

In the Apollo program, astronauts wore custom tailored suits and had the opportunity to undergo multiple fit tests to ensure that their suits fit. To quote David Scott of Apollo 15, "So they felt that if you had a proper fit, then you had better mobility." [1] The importance of a tailored fit is also mentioned in the Mission Report for Apollo 16: "The suits are custom fitted and, by necessity, must be tight to achieve good mobility." [2]

The combination of custom tailoring and some adjustability in the suit allowed the Apollo astronauts to correct minor sizing issues in flight, such as a case where the legs of a pressure garment were too short, leading to discomfort [3].

However, some fit problems remained, even with custom fit suits. For example, Astronaut Edwin Aldrin had large biceps which allegedly interfered with the arm bearings on his suit, and prevented his fingers from seating correctly in his gloves when he bent his arms [4].

Gloves were also an issue, as discussed by the crew of Apollo 15 in the technical debriefing for that flight [5]. Crewmember David Scott brings up a common problem with space suit fit: the compromise between arm length and glove mobility. If a suit is sized to fit the crewmember when their arms are outstretched, the fingers are forced back out of the gloves when they pull their arms close to the chest. If, on the other hand, the suit is sized for the fingers to be snug when working close to the chest, the fingertips will press against the glove when the arms are at other postures. As a result, Scott had his suit arm length adjusted to keep his fingers in the gloves, and accepted the sore and painful fingertips that resulted from this fit.

\subsection{Modularity: The Shuttle Extravehicular Mobility Unit}

The architects of the space shuttle program abandoned the custom fit suits of Apollo in the interests of improving manufacturing efficiency, reducing cost and allowing easier maintenance and resizing. The designers of the space shuttle Extravehicular Mobility Unit (EMU) were tasked with developing a set of modular suits to fit a population that could include everyone from a $5^{\text {th }}$ percentile female to a $95^{\text {th }}$ percentile male [6]. However, as the suits were developed the number of sizes of Hard Upper Torso (HUT), a major suit component, was cut back. Because only the M, L and XL HUT sizes were developed, many smaller women cannot wear the current shuttle EMU.

The compromise between arm length and glove mobility also continues in the shuttle program, where fingertip pressure has been indicated as a possible source for fingertip pain and fingernail delamination [7].

\subsection{Standard Sizes: The Advanced Crew Escape Suit}

The Advanced Crew Escape Suit (ACES) is similar to pressure suits developed for the US Air Force, and has a similar sizing scheme based on the height and weight of the wearer. However, the pressure suit was sized for a seated pilot in an aircraft, and not for the walking, running or climbing that a shuttle crewmember may perform during training. As a result, many crewmembers allegedly choose to wear a size that is larger than the size recommended for them by the sizing scheme [8]. 


\subsection{Consequences of Poor Suit Fit}

The cost of a poor suit fit, as suggested in the previous sections, can include wearer discomfort and a reduction in mobility. A suboptimal suit fit can also increase the effort that a wearer must exert to move in the suit, since their joints are not lined up with the suit joints. As described by Menendez and colleagues in their 1993 paper, the instantaneous centers of rotation of the human and space suit joints should be co-located to minimize the energy needed to move [9] For instance, a wearer is likely to move less efficiently if the suit's knee joint is several inches above or below his own knee.

Also, a poorly fit suit has the potential to actually impinge on the wearer during motion and lead to a reduction in mobility and risk of injury. In a 2003 report on shoulder injuries in the space shuttle EMU, it was suggested that the scye openings on a poorly sized hard upper torso could restrict shoulder motion and lead to injury [10].

\section{Improving Fit: What Is Fit?}

When attempting to achieve a good space suit fit, it seems reasonable to examine solutions that have been developed to fit clothing and gear in the past. The problem of fit has often been solved by trial and error, and through time consuming and expensive tailoring processes. More recently, attempts have been made to find more efficient ways of fitting people. However, if fit is to be optimized it must first be defined and understood.

\subsection{Fit: An Objective Measure?}

Fit is often considered to be a subjective measurement of garment quality, and is expected to vary from person to person, and sometimes even between the same person on different days. However, experts would argue that a garment's "fit" is merely an indication of the garment's tailoring, or the complex relationship of the garment dimensions to the dimensions of the wearer [11]. If a person claims that their clothing does not fit them, they are indicating dissatisfaction with the garment's tailoring in one or more areas. This difference between garment and wearer dimensions is called "ease".

A garment that has too much or too little ease in any location can lead to poor fit and dissatisfaction of the wearer. To complicate matters, a garment's dimensions do not only have to accommodate a stationary wearer; there must be enough additional material to provide for extreme motions such as kneeling and reaching overhead [12]. The problem of sufficient material, or 'run length', becomes even more complex for a one-piece garment such as a coverall, where addition of material in one area can affect the fit of the entire garment [13]. Making an item oversized can solve some problems, but excess material causes its own issues. For instance, in their 2007 paper, $\mathrm{Ng}$ et al. describe the problem of fit in the shoulder area [11]. If the scye area (the arm opening) of a garment is too wide or too far from the underarm it can restrict the wearer as much as a sleeve opening that is too tight.

\section{Improving Fit: Sizing Systems}

Admittedly, there is a subjective component to fit and comfort. However, gross approximations of correct size should be made before fit is fine tuned on an individual 
basis. This approximation is achieved through the development of a standardized sizing system that will attribute a size to an individual.

\subsection{Traditional Sizing Systems and Limitations}

Sizing systems are often developed using the anthropometry from a sample of intended wearers. For example, a US Air Force flight suit sizing system [15] was based on a 1967 survey of 2420 men. Individuals in the sample are then generally categorized based on a pair of so-called key dimensions, which are easy to measure and which are assumed to be highly correlated with other dimensions. For example, a sizing system may be based on a combination of height and weight.

Once the individuals in the sample have been split up into categories based on intervals of key dimensions, each category is examined individually. Anthropometry from individuals in a given size category is used to develop regression equations, which will predict minor dimensions like chest breadth.

The use of key dimensions allows easy size selection, since a table of two dimensions can be consulted when assigning a piece of equipment to an individual. However, this method assumes that an individual's shape and size can be predicted accurately using two basic dimensions such as height and weight - an assumption that is not necessarily accurate. This limitation becomes obvious as the number of sizes increases. Increasing the number of sizes would initially seem to improve fit, but can actually cause more fit problems because peoples' minor dimensions are allowed less variability within a size.

The issues associated with a simplistic sizing scheme are a symptom of the large amount of variation in peoples' shapes and sizes, their so-called 'somatotypes.' For instance, for a given height and weight you might find people with long torsos and short legs, or short torsos and long legs. A tall muscular person could potentially weigh the same as a tall obese person, but have a very different shape.

\subsection{Sizing for Men and Women}

The problem of fitting a wide range of sizes and shapes of people is further complicated if both men and women must be fit with the same sizing system. Issues have arisen in the past when military organizations have attempted to accommodate women using systems that have been designed for men.

Some groups initially assumed that women could fit in the same sizes as small men - or at worst, that some of the men's sizes would have to be scaled down proportionately to fit women [16]. However, problems arise due to the very different proportions between men and women. For the same height and weight, women can have significantly wider hips and narrower shoulders than men. If, for example, a onepiece coverall designed for a man is meant to fit at the shoulders and the hips, then one of these fit areas is likely to be compromised for a woman. She has to choose a size that fits over her hips, likely leading to the coverall's shoulders being too wide for her frame.

Several approaches have been taken towards fitting both men and women with the same gear. These methods are summarized in Figure 1. 


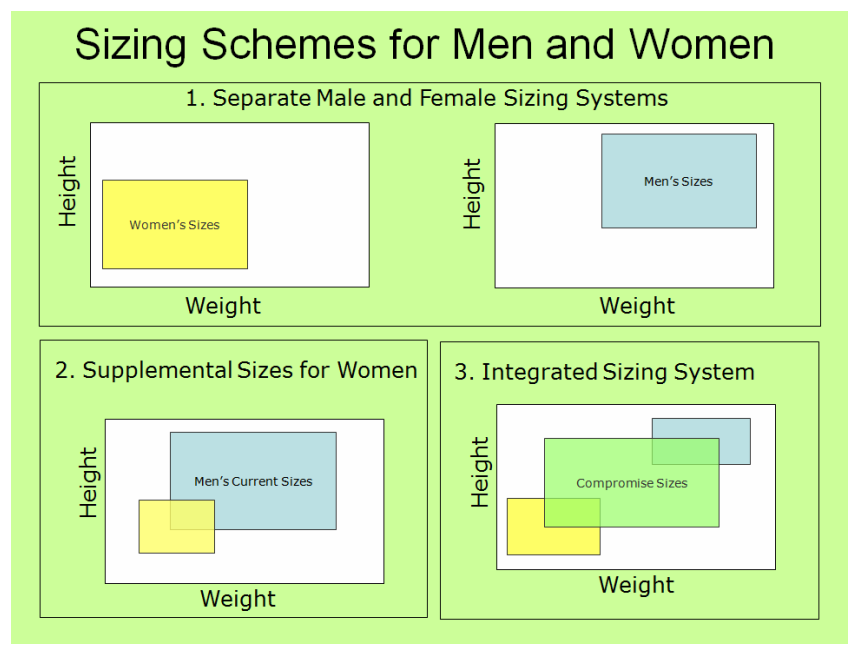

Fig. 1. Sizing Schemes for Men and Women

For systems such as a naval uniform where a tailored fit is considered important, a female-only sizing scheme can be created (section 1 of Figure 1) as described in a 1991 paper by Armstrong Laboratory [17]. For types of equipment where fit may not be as essential, a few extra sizes can be designed for poorly fit women, with the assumption that most women can wear men's sizes with an acceptable decrement in fit (section 2 of the figure). Additionally, in at least one case, a theoretical integrated sizing system was developed (shown in section 3 of the figure).

An example of integrated sizing system for the US army Battle Dress Uniform (BDU) is described in a 1981 report from the Natick Research and Development Lab [18]. This integrated system optimizes the smallest BDU sizes for women, optimizes the largest sizes for men, and forms a compromise with the intermediate sizes that both men and women would wear. For instance, an extra-extra-small extra-short pair of pants might be rarely worn by men - in which case, the pants could be designed for a woman's generally larger hips and smaller waist. A more intermediate size might still accommodate a woman's hips, but provide for larger waists. The compromise in this size might lead to loose hips on the men and loose waists on the women, but within an acceptable range.

\subsection{Better Sizing Systems: Multivariate Methods}

To solve the oversimplification problem associated with sizing systems which rely on paired key dimensions like height and weight, some groups have attempted to use multivariate methods to develop sizing schemes. Using techniques such as principal component analysis and cluster analysis, sizing system designers can account for the wide variation in human shape by grouping together people of similar somatotype.

For example, Zehner et al. developed a sizing system that used principal component analysis to group a selection of anthropometry from a sample population [19]. This analysis led to a component contrasting limb to torso size and a component representing overall body size. These two components could then be used to represent a 
wide variety of body shapes and sizes. Designers can pick a body type, for example a small individual with small torso and small limbs, and then look at the anthropometry for a person in the database whose values for the two components reflect this shape. This method allows designers to base their design on the anthropometry of an actual person in a given category, and to have greater confidence that their design will accommodate people of varying sizes and shapes.

\subsection{Sizing Systems for Space Suits}

As touched on previously, a sizing system for the next generation of NASA suits will have several additional layers of complexity beyond sizing systems developed for clothing. For one, a space suit that is sized for unpressurized use must also fit when pressurized. Also, space suits must provide enough adjustability to allow for the elongation of the human spine in microgravity, an elongation that may be as much as $3 \%$ of a wearer's stature [20]. In other words, if a suit is sized precisely on the ground while unpressurized, the fit may change in space and when the suit is pressurized. Also, cost and mass restrictions will govern the number of suits created and flown by the space program. This limited number of suits will be required to fit an incredibly diverse population that can comprise anyone from a theoretical $1^{\text {st }}$ percentile female to a $99^{\text {th }}$ percentile male. This population also has the potential to vary significantly in shape, and could include a tall man with short legs as easily as a short woman with long legs.

The complexity of space suit fit means that multivariate methods will likely have to be used to develop a sizing system with sizes optimized for a future astronaut population, with enough adjustability to account for variations in the size and shape of its potential wearers, as well as adjustments for pressurized vs. unpressurized fit, accommodation for spinal elongation, and personal preference of the wearer.

\section{From Anthropometry to Fit}

The dimensions of the wearer provide only a starting point for the design. If clothing were made to fit a human's anthropometry, with no additional slack, it would be skin tight. Therefore, using tailoring techniques that have been developed over centuries, designers add ease allowances and seam allowances to develop a final garment [16]. However, this initial ease allowance may not provide enough run length to allow for extreme motions. To evaluate the amount of ease in a garment, fit checks are performed.

\subsection{Subjective Fit Checks}

A fit check can be as simple as putting on the garment and performing some basic motions, while an expert assessor evaluates the fit using a checklist or questionnaire. An example of this type of fit check is documented in a 1995 paper on flight suit fit by Crist et al. [21].

\subsection{Objective Fit Checks: Range of Motion Testing}

Several studies have evaluated the range of motion of a subject while varying the amount of ease. This method allows a quantitative measure of how the subject's 
restriction varies with change in the shape of the garment. For example, Huck, Maganga and Kim controlled the shape and size of a custom-made garment, except for one location where they increased or decreased the amount of ease [13]. In other studies, a subject is first provided with a garment in their recommended size based on a sizing chart, as in a study by Adams et al. published in 1995 [22]. An evaluation protocol is performed in the recommended garment size, and then in garments that are longer or shorter, larger or smaller according to the sizing chart. The protocol typically involves a series of predetermined motions or exercises meant to take up any slack in the garment. One such posture, suggested by Crow and Dewar in their 1986 paper on stress in clothing seams, involves squatting and lifting the arms over the head [23]. In a one piece garment such as a coverall, the squatting motion takes up a lot of the ease, leaving very little slack when a subject then reaches their arms over their head (see Figure 2, from an unpublished pilot study).

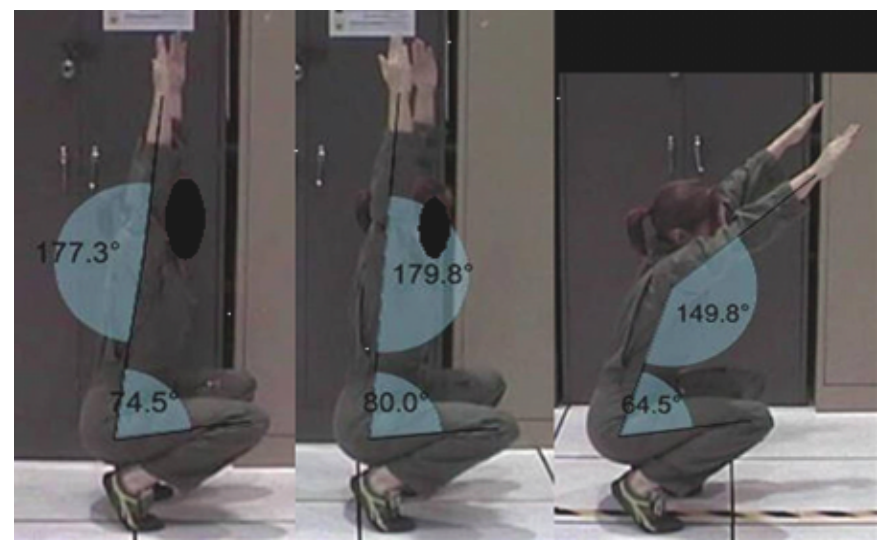

Fig. 2. Example: Posture while crouching and reaching overhead in oversized (Left), appropriately Sized (Center) and undersized (Right) Flight Suits

The subject in the figure is wearing three different sizes of flight suit: a coverall that is too large, a coverall that is appropriately sized, and a coverall that is too small. The oversized and appropriately sized coverall were approximately equivalent, but an obvious restriction in motion was observed for the smallest flight suit.

In another study, $\mathrm{Ng}$ et al. developed a model of the interaction between several garment dimensions and the range of motion of the arm [11]. By optimizing the location of the underarm point, they found that they could find an approximate solution for a sleeve with the minimum amount of fabric to provide a given range of arm motion.

\subsection{Applying Fit Testing to Suits}

Because the fit of a suit is crucial for comfort, operability and safety, and because a poor suit fit is likely to cause a decrement in performance, testing should be completed to evaluate a suit's fit. Objective fit testing during the design of a space suit architecture could aid designers in assessing how well they are fitting their target population, and could potentially indicate where slack must be taken out or added, or 
where additional adjustability is needed. Although the flight suit study suggested that range of motion can be improved by providing additional slack, there are cases where too much clearance causes issues, as in the case of the current space shuttle EMU, where the issue is not so much fabric slack, but the size of the hard upper torso.

Without performing fit testing, it is difficult to assess the unknown impact of suit fit while evaluating the space suit or while evaluating a system that will interact with the suit. If a subject is wearing a suit that has a marginal fit, they may be exerting more effort to perform a given task than a subject wearing a suit in their proper size. The suit's suboptimal fit could lead to an undeservedly poor evaluation of the suit, or of the system being tested.

\section{Conclusion and Future Work}

As the next generation of space suits are developed for NASA's Constellation program, steps should be taken to ensure that the suits will adequately fit their target population. This task can include the development of a sizing system that optimizes the number of space suit sizes and their required adjustability, without compromising accommodation for any sector of the population. As suit prototypes are developed, objective fit checks can evaluate how well the new suit is fitting a sample of its population and help to indicate problem areas for fit. Compromises will have to be made to accommodate both male and female wearers of widely varying size and shape without unduly reducing mobility or decreasing efficiency for any one wearer. An acceptable suit fit will also allow more realistic assessment of not only the suit, but also of systems that interact with the suit during man in the loop tests.

\section{Acknowledgements}

The writers of this paper would like to acknowledge Amy Ross and Terry Hill of the Pressure Garment group at NASA, as well as Scott Cupples and Brian Johnson of the EVA Project Office, for their funding and support.

\section{References}

1. Jones, E.M.: Apollo Lunar Surface Journal, http: //history.nasa.gov/alsj/a15/a15.spur.html

2. Jones, E.M.: Apollo Lunar Surface Journal: Apollo 16 Mission Report Online: http://history.nasa.gov/alsj/a16/A16_MissionReport.pdf

3. Jones, E.M.: Apollo Lunar Surface Journal: Apollo 12 Mission Report Online: http: / / www.hq.nasa.gov/alsj/a12/A12_MissionReport.pdf

4. Jones, E.M.: Apollo Lunar Surface Journal: Apollo 11 Post-Flight Report on Suits / PLSSs / etc (Preliminary Version), http: / /www.hq.nasa.gov/alsj/a11/A11CSD.html

5. Jones, E.M.: Apollo Lunar Surface Journal: Apollo 15 Technical Debrief, http://history.nasa.gov/alsj/a15/a15-techdebrief.pdf 
6. Currie, N.J., Graziosi, D.: Space Suit Design Enhancements to Improve Size Accommodation and Mobility. In: Proceedings of the Human Factors and Ergonomics Society $47^{\text {th }}$ Annual Meeting 2003 (2003)

7. Strauss, S.: DO, MPH. Extravehicular Mobility Unit Training Suit Symptom Study Report. NASA/TP-2004-212075

8. Transcript, Jean Alexander Oral History, Houston, TX by Kevin Rusnak, Johnson Space Center Oral History Project, June 23 (1998)

9. Menendez, V., Labourdette, X., and Baez, J.M.: Performance of EVA Suit Mobility Joints Influence of Driving Parameters. In: 23rd International Conference on Environmental Systems, SAE Paper 932098 (July 1993)

10. Williams, D.R., Johnson, B.J.: EMU Shoulder Injury Tiger Team Report. NASA/TM2003-212058

11. Ng, R., et al.: Single Parameter Model of Minimal Surface Construction for Dynamic Garment Pattern Design. Journal of Information and Computing Science 2(2), 145-152 (2007)

12. Adams, P., Slocum, A., Herrin, G.: An Approach for Predicting Garment Effects on Range-of-Motion Based on Measurement of Garment Ease. In: Proceedings of the Second International Symposium on Consumer Environmental Issues: Safety, Health, Chemicals and Textiles in the Near Environment, pp. 216-229 (1992)

13. Huck, J., Maganga, O., Kim, Y.: Protective Overalls: Evaluation of Garment Design and Fit. International Journal of Clothing Science and Technology 9(1), 45-61 (1997)

14. Ng, R., et al.: Single Parameter Model of Minimal Surface Construction for Dynamic Garment Pattern Design. Journal of Information and Computing Science 2(2), 145-152 (2007)

15. Alexander, M., McConville, J., Tebbetts, I.: Revised Height/Weight Sizing Programs for Men's Protective Flight Garments. Technical Report (AMRL-TR-79-28) (AD A070 732). Aerospace Medical Research Laboratory, Wright-Patterson Air Force Base, OH (1979)

16. Robinette, K.: Flight Suit Sizes for Women. (AD A321 200) Armstrong Laboratory, Brooks Air Force Base, TX (1996)

17. Robinette, K.M., et al.: Development of Sizing Systems for Navy Women's Uniforms (U). AL-TR-1991-0117

18. McConville, J., Robinette, K., White, R.: An Investigation of Integrated Sizing for US Army Men and Women. Anthropology Natick/TR-81/033 (AD A109 406)

19. Zehner, G.F., Meindl, R.S., Hudson, J.A.: A Multivariate Anthropometric Method for Crew Station Design: Abridged, AL-TR-1992-0164. Armstrong Laboratory, WrightPatterson Air Force Base (1992) (AD A274 588)

20. Stoycos, L.E., Klute, G.K.: Anthropometric Data from Launch and Entry Suited Test Subjects for the Design of a Recumbent Seating System. NASA Technical Memorandum 104769 (1993)

21. Crist, J., Gross, M., Robinette, K., Altenau, M.: Fit Evaluation of Two Aircrew Coveralls. AL/CF-TR-1995-0053, Armstrong Laboratory, Air Force Material Command, Wright Patterson Air Force Base, OH (1995)

22. Adams, P., Keyserling, W.M.: The Effect of Size and Fabric Weight of Protective Coveralls on Range of Gross Body Motions. American Industrial Hygiene Association Journal 56(4), 333-340 (1995)

23. Crow, R.M., Dewar, M.M.: Stresses in Clothing as Related to Seam Strength. Textile Research Journal 56(8), 467-473 (1986) 